\title{
Commentary \\ Collagen-related biomarkers in severe sepsis: a big stretch?
} Gavin Morrison ${ }^{1}$ and Douglas D Fraser ${ }^{1,2,3}$

\author{
${ }^{1}$ Critical Care Medicine and Paediatrics, University of Western Ontario, London, ON, N6A 5W9, Canada \\ ${ }^{2}$ Children's Health Research Institute and Centre for Critical IIIness Research, London, ON, N6C 2V5, Canada \\ ${ }^{3}$ Translational Research Centre, London, ON, N6C 2V5, Canada
}

Corresponding author: Douglas D Fraser, fraserd@lhsc.on.ca

Published: 2 June 2009

Critical Care 2009, 13:154 (doi:10.1186/cc7879)

This article is online at http://ccforum.com/content/13/3/154

(C) 2009 BioMed Central Ltd

See related research by Gäddnäs et al., http://ccforum.com/content/13/2/R53

\begin{abstract}
Biomedical scientists are aggressively investigating biomarkers of disease and injury. The rationale for identifying biomarkers during pathological states, such as severe sepsis, is to improve clinical prognostication and stratify therapeutic interventions for optimal recovery. An added benefit of biomarker studies is knowledge genesis on pathophysiological mechanisms, critical information that provides a basis for hypothesis-driven research. Unfortunately, biomarkers rarely alter our clinical approach in severe sepsis as they are often non-specific, lack adequate sensitivity and/or are difficult to measure and interpret accurately. Given the complexity and heterogeneity of severe sepsis, and the unique genetically derived susceptibilities of individuals, it is highly unlikely that one or even a handful of biomarkers will provide adequate biomedical information for clinical guidance. Thus, biomarkers will ultimately alter clinical decision making only once a panel of promising biomarkers is identified, maximizing sensitivity and specificity, and then adequately scrutinized with quantitative scoring methods over large populations of patients.
\end{abstract}

Severe sepsis is a leading cause of morbidity and mortality. Despite increased understanding of its pathophysiology, our ability to prognosticate in individual patients with severe sepsis is poor. Thus, there are ongoing investigations to discover novel biomarkers to complement our clinical ability to predict disease evolution and stratify therapies. Gäddnäs and colleagues [1] report in a recent issue of Critical Care that the utilization of collagen metabolism biomarker levels retrospectively exhibited correlation with disease severity and differentiated between survivors and non-survivors in a cohort of patients with severe sepsis. The authors postulate that the products of collagen metabolism may have a role as biomarkers in severe sepsis, but is measurement of these collagen-related biomarkers likely to provide meaningful clinical guidance?
Collagens comprise approximately 30\% of the body's protein content and provide much of the structural strength and elasticity of human tissue [2]. Type I and III collagen fibers are derived from procollagen precursor molecules from which amino-terminal propeptides are cleaved to generate the amino-terminal propeptides of type I procollagen (PINP) and type III procollagen (PIIINP). Type I collagen is widely distributed and PINP has been utilized as an index of type I collagen synthesis in tissues. Type III collagen is found in skin, blood vessel walls and hollow viscera. PIIINP is most commonly utilized as a marker of type III collagen synthesis in early wound repair, but increased concentrations of PIIINP have also been described in various disease states. The carboxy-terminal telopeptide of type I collagen (ICTP) is a product of type I collagen elimination. As nicely demonstrated by Gäddnäs and colleagues [1], PINP, PIIINP and ICTP were all elevated in patients with severe sepsis, indicating significant collagen metabolism, with PINP and PIIINP levels correlating with disease severity.

While intriguing, the results of this severe sepsis biomarker study by Gäddnäs and colleagues [1] are likely contaminated by co-morbidities and administered therapies. For example, increased PIIINP levels have been measured in the serum of patients with hepatic cirrhosis and acute respiratory distress syndrome, and in the bronchoalveolar lavage fluid of patients with sarcoidosis and fibrosing alveolitis. Of more direct relevance, ischaemic heart disease, chronic heart failure, diabetes and chronic obstructive airway disease (which represented the co-morbidities in $61 \%$ of the patients with severe sepsis [1]) are also associated with altered collagen metabolism. Furthermore, therapies such as norepinephrine (which was administered to $86 \%$ of the severe sepsis

$\mathrm{ICTP}=$ carboxy-terminal telopeptide of type I collagen; IL = interleukin; MMP = matrix metalloproteinase; PINP = amino-terminal propeptide of type I procollagen; PIIINP = amino-terminal propeptide of type III procollagen; TIMP = tissue inhibitor of matrix metalloproteinase. 
patients [1]) produce increases in type I and III collagen within the extracellular matrix of the heart [3]. Given these confounding influences, there is some difficulty in teasing out the effect of severe sepsis per se, a problem exacerbated by a limited sample population. The investigators appropriately recognize that matching of chronic disease states would enhance the study [1], but this omission might be of more significance than a simple study design weakness.

Fibrosis is an end stage process consisting primarily of collagen deposition [4]. Extracellular matrix remodeling is, however, dynamic, altering in character as the disease process develops. Collagen metabolism is largely regulated by proteases, particularly a class of enzymes referred to as the matrix metalloproteinases (MMPs) and their endogenous tissue inhibitors (TIMPs) [5]. MMPs and TIMPs not only regulate collagen turnover, but are also the effectors for the action of a number of interleukins and growth factors, and may represent a more appropriate measure of the state of tissue remodeling in severe sepsis than collagen-related factors. For example, a recent study reported elevated levels of MMP-9, TIMP-2 and TIMP-1 in severe sepsis [6], with TIMP-1 identified as having some potential to serve as a useful biomarker in predicting the clinical outcome of patients with severe sepsis.

An intriguing observation reported by Gäddnäs and colleagues [1] is the persistence of increased levels of PIIINP and PINP, suggesting that severe sepsis continues to cause metabolic disequilibrium long after clinical recovery. Severe sepsis might initiate a sustained inflammatory response, associated with ongoing collagen metabolism, thereby underlying the poor long-term outcome in adult survivors [7]. While an in-hospital mortality of $51 \%$ was reported in 153 adults with sepsis, the post-hospital discharge mortality at 6 months and 1 year was $68 \%$ and $79 \%$, respectively [7]. The continued evidence of disordered collagen metabolism [1], presumably in the absence of treatment effects, may render the byproducts of collagen production more useful biomarkers of a chronic dysfunction rather than acute phase reactants. Indeed, evidence demonstrates that disordered collagen metabolism results in significant disruption of tissue structure and function due either to collagen loss or excessive deposition.

An ideal severe sepsis biomarker must be specifically linked to the disease itself and to a therapeutic decision, not only its differential expression in severe sepsis patients or even its association with adverse outcome [8]. Collagen metabolism biomarkers are plausible prognostic agents, as reported by Gäddnäs and colleagues [1], but only in the context of a larger panel of biomarkers [9]. Other promising severe sepsis biomarkers, which might eventually be combined with collagen-related markers, include IL-6, IL-8, IL-10, C-reactive protein, procalcitonin, MMP-9 and TIMP-1 [6,10-13]. Once an ideal panel of biomarkers is determined, relatively rapid biomarker assays using multiplex [14] and antibody microarray [15] technology may be widely available in the near future and yield critical severe sepsis prognostic information in the form of a patient-specific biomarker profile.

\section{Competing interests}

The authors declare that they have no competing interests.

\section{References}

1. Gäddnäs FP, Koskela M, Koivukangas V, Risteli J, Oikarinen A, Laurila JJ, Saarnio J, Ala-Kokko TI: Markers of collagen synthesis and degradation are increased in serum in severe sepsis: alongitudinal study of 44 patients. Crit Care 2009, 13:R53.

2. Prockop DJ, Kivirikko KI, Tuderman L, Guzman NA: The biosynthesis of collagen and its disorders (first of two parts). $N$ Engl J Med 1979, 301:13-23.

3. Briest W, Rassler B, Deten A, Zimmer HG: Norepinephrineinduced cardiac hypertrophy and fibrosis are not due to mast cell degranulation. Mol Cell Biochem 2003, 252:229-237.

4. Prockop DJ, Kivirikko KI, Tuderman L, Guzman NA: The biosynthesis of collagen and its disorders (second of two parts). $N$ Engl J Med 1979, 301:77-85.

5. Chow AK, Cena J, Schulz R: Acute actions and novel targets of matrix metalloproteinases in the heart and vasculature. $\mathrm{Br} J$ Pharmacol 2007, 152:189-205.

6. Hoffmann U, Bertsch T, Dvortsak E, Liebetrau C, Lang S, Liebe V, Huhle G, Borggrefe M, Brueckmann M: Matrix-metalloproteinases and their inhibitors are elevated in severe sepsis: prognostic value of TIMP-1 in severe sepsis. Scand J Infect Dis 2006, 38:867-872.

7. Sasse KC, Nauenberg E, Long A, Anton B, Tucker HJ, Hu TW: Long-term survival after intensive care unit admission with sepsis. Crit Care Med 1995, 23:1040-1047.

8. Marshall JC: Biomarkers of sepsis. Curr Infect Dis Rep 2006, 8: 351-357

9. Shapiro NI, Trzeciak S, Hollander JE, Birkhahn R, Otero R, Osborn TM, Moretti E, Nguyen HB, Gunnerson KJ, Milzman D, Gaieski DF, Goyal M, Cairns CB, Ngo L, Rivers EP: A prospective, multicenter derivation of a biomarker panel to assess risk of organ dysfunction, shock, and death in emergency department patients with suspected sepsis. Crit Care Med 2009, 37:96104.

10. Giamarellos-Bourboulis EJ, Mega A, Grecka P, Scarpa N, Koratzanis G, Thomopoulos G, Giamarellou H: Procalcitonin: a marker to clearly differentiate systemic inflammatory response syndrome and sepsis in the critically ill patient? Intensive Care Med 2002, 28:1351-1356.

11. Harbarth S, Holeckova K, Froidevaux C, Pittet D, Ricou B, Grau GE, Vadas L, Pugin J: Diagnostic value of procalcitonin, interleukin-6, and interleukin-8 in critically ill patients admitted with suspected sepsis. Am J Respir Crit Care Med 2001, 164: 396-402.

12. Claeys R, Vinken S, Spapen H, ver Elst K, Decochez K, Huyghens $\mathrm{L}$, Gorus FK: Plasma procalcitonin and C-reactive protein in acute septic shock: clinical and biological correlates. Crit Care Med 2002, 30:757-762.

13. Kinasewitz GT, Yan SB, Basson B, Comp P, Russell JA, Cariou A, Um SL, Utterback B, Laterre PF, Dhainaut JF: Universal changes in biomarkers of coagulation and inflammation occur in patients with severe sepsis, regardless of causative microorganism [ISRCTN74215569]. Crit Care 2004, 8:R82-90.

14. Fulton RJ, McDade RL, Smith PL, Kienker LJ, Kettman JR Jr: Advanced multiplexed analysis with the FlowMetrix system. Clin Chem 1997, 43:1749-1756.

15. Garcia BH, 2nd, Hargrave A, Morgan A, Kilmer G, Hommema E, Nahrahari J, Webb B, Wiese R: Antibody microarray analysis of inflammatory mediator release by human leukemia T-cells and human non small cell lung cancer cells. J Biomol Tech 2007, 18:245-251. 